\title{
Concreto autoadensável com baixo consumo de cimento: impacto da redução do consumo de cimento na resistência à compressão
}

\section{Self-compacting concrete with low cement consumption: impact of the reduction of cement consumption on the compressive strength}

\footnotetext{
${ }^{1}$ Professor do UNASP e Doutorando do Programa de pós-graduação de Engenharia Civil da Universidade Federal de São Carlos (UFSCar), Caixa Postal 11; CEP 13165-000, Engenheiro Coelho, SP, Brasil.

e-mail:lucas.barboza@unasp.edu.br

${ }^{2}$ Professor Doutor do Departamento de Engenharia Civil, Universidade Federal de São Carlos (UFSCar), CEP 13565905, São Carlos, SP, Brasil.

e-mail: almeidafilho@ufscar.br
}

\section{RESUMO}

A consolidação do concreto como segundo material mais consumido no mundo tem instigado a busca de soluções para reduzir o consumo de clínquer e as emissões de $\mathrm{CO}_{2}$. Visando o fornecimento de uma contribuição para a sustentabilidade ambiental e da indústria de cimento, esta pesquisa busca analisar a viabilidade econômica do concreto autoadensável (CAA) com baixo consumo de cimento. Por intermédio da aplicação do método de dosagem de Gomes (2002) para o CAA, foi realizado um estudo da otimização do esqueleto granular e da composição da pasta, sendo aferido reduções de até $25 \%$ do consumo de cimento em relação ao CAA de referência. No que tange aos índices de consumo relativo de cimento e de custo unitário por MPa desenvolvido em $1 \mathrm{~m}^{3}$ de concreto, foi verificado que os CAAs com baixo consumo de cimento apresentaram reduções de até $34,75 \%$ e $16,83 \%$, respectivamente, ao ser comparado com o CAA de referência. A maior efetividade na redução do índice de consumo relativo a resistência mecânica à compressão do que no consumo de cimento por $\mathrm{m}^{3}$ de concreto observado, se deve a constatação de maiores patamares de resistência mecânica à compressão para a composição com menor volume de pasta e de maior proximidade ao índice ótimo de vazios encontrado na etapa do empacotamento de agregados. Tal resultado, demonstra indícios de que mesmo nas circunstâncias de dosagens com volume de pasta inferiores, a resistência mecânica à compressão pode ser aumentada, comprovando desta maneira a superioridade técnica e financeira do CAA com baixo consumo de cimento em relação as dosagens convencionais de CAA, além dos ganhos em sustentabilidade obtidos pela redução do consumo de clínquer e das emissões de $\mathrm{CO}_{2}$ inerentes a menor quantidade de massa de cimento por $\mathrm{m}^{3}$ de concreto.

Palavras-chave: Concreto autoadensável, consumo de cimento, emissões de $\mathrm{CO}_{2}$, resistência à compressão.

\section{ABSTRACT}

The consolidation of concrete as the second most consumed material in the world has instigated the search for solutions to reduce clinker consumption and $\mathrm{CO}_{2}$ emissions. Aiming to provide a contribution to environmental sustainability and the cement industry, this research seeks to analyze the economic viability of self-compacting concrete (SCC) with low cement consumption. A study of the optimization of the granular skeleton and the composition of the paste was carried out using the Gomes (2002) dosing method for the SCC. Reductions of up to $25 \%$ of the cement consumption were measured in relation to the reference SCC. Regarding the relative cement consumption and unit cost indexes per $\mathrm{MPa}$ developed in $1 \mathrm{~m}^{3}$ of concrete, it was verified that the SCC's with low cement consumption showed reductions of up to $34.75 \%$ and $16.83 \%$, 
respectively, while being compared to the reference SCC. The greater effectiveness in the reduction of the consumption relative to the mechanical resistance to compression than in the consumption of cement per cubic meter of concrete observed, is due to the observation of higher levels of mechanical resistance to compression for the composition with lower paste volume and higher proximity to the optimal void index found in the aggregate packaging stage. This result shows that even in the lower dosage circumstances, the mechanical compressive strength can be increased, thus demonstrating the technical and financial superiority of the SCC with low cement consumption compared to conventional SCC dosages, in addition to the sustainability gains obtained by the reduction of the clinker consumption and the $\mathrm{CO}_{2}$ emissions inherent to the lower amount of cement mass per cubic meter of concrete.

Keywords: Self-compacting concrete, cement consumption, $\mathrm{CO}_{2}$ emissions, compressive strength.

\section{INTRODUÇÃO}

Com o crescente avanço tecnológico dos materiais e das técnicas da construção civil, misturas de concretos especiais surgiram na tentativa de superar as deficiências apresentadas pelos concretos convencionais e atender aos requisitos da sustentabilidade [1]. Fundamentado neste conceito, o Concreto Autoadensável com Baixo Consumo de Cimento (CAABCC) pode oferecer vantagens únicas e desempenho mecânico diferenciado, provando que não é necessário um alto consumo de cimento para obtenção de resistência e durabilidade.

Pode-se considerar o CAABCC como uma evolução tecnológica dos concretos tradicionais, oriundo da pesquisa aplicada e resultado da combinação de materiais comuns e de fácil aquisição no mercado da construção civil, e de dois novos componentes: as adições minerais e os aditivos químicos, no qual contribuem para redução da porosidade e da permeabilidade do concreto.

Por se tratar de uma representação da evolução tecnológica do concreto, alguns fatores importantes devem ser considerados para que seja possível a produção do mesmo, como controle da temperatura e umidade (tanto do ambiente como a dos materiais), equipamentos de mistura e transportes mais eficientes, refinamento dos processos executivos para produção do concreto e conhecimento da interação entre os materiais constituintes.

As dúvidas pertinentes sobre a eficiência mecânica de concretos com baixo consumo de cimento e a sua projeção no futuro justificam a avaliação da resistência destes concretos. Possivelmente novos limites para consumos mínimos de cimento possam ser estabelecidos, não apenas adotando o parâmetro de resistência, mas, sobretudo, de desempenho. $\mathrm{O}$ acompanhamento da viabilidade técnica e da viabilidade econômica deve ser simultâneo. Um bom desempenho financeiro não se restringe apenas à diminuição dos custos empregados inicialmente, mas, também à obtenção de bom desempenho e durabilidade para que ocorra a redução dos custos de manutenção e, assim, gerando um aumento de vida útil [2].

REBMANN [2] apontou que comumente a obtenção de um concreto de alta resistência e durável, é associada a um alto consumo de cimento Portland, no qual, este procedimento pode gerar alguns inconvenientes no desempenho de uma estrutura, como uma maior tendência de desenvolver fissuras decorrentes da retração térmica e química.

De acordo com MEHTA e MONTEIRO [3], entre os materiais que compõem o concreto, o cimento Portland é o que demanda maior custo e consumo energético para ser produzido, podendo emitir em torno de 1 tonelada de $\mathrm{CO}_{2}$ (Dióxido de carbono) para cada $1 \mathrm{t}$ de clínquer produzido, o que representa cerca de $90 \%$ da emissão de $\mathrm{CO}_{2}$ da indústria do concreto. Com um consumo crescente ao longo dos anos, tem sido considerado como um dos principais contribuintes na emissão de $\mathrm{CO}_{2}$ na atmosfera [4].

O desafio hoje na construção civil é torná-lo (CAABCC) uma opção viável para obras do cotidiano, fazendo com que o desenvolvimento que se obteve desses materiais em laboratório atravesse a barreira do mercado, tornando-se, assim, uma opção viável para as construções. Em resumo, o CAABCC tem um forte potencial para revitalizar a infraestrutura das edificações, e das novas infraestruturas, tornando-as resistente e de longa duração.

A tecnologia dos concretos estruturais, apoiada em princípios científicos, tem dado sua contribuição para este desafio. O desenvolvimento de concretos com o uso intensivo de superplastificantes, uso de adições de minerais provenientes de resíduos industriais e o empacotamento de partículas tem permitido a redução do consumo de aglomerantes, sem prejuízo na durabilidade. Vale mencionar que os recursos tecnológicos já existem, cabendo apenas à indústria da construção civil a sua popularização e aplicação [1].

Tratando do concreto autoadensável, exige-se consumos de finos e cimento superiores ao concreto convencional, para que o mesmo apresente as seguintes propriedades: deformabilidade, resistência à segregação, habilidade de passagem e velocidade de fluidez [5]. 
De acordo com GOMES [6], em relação ao consumo de finos, o mesmo é cerca de 400 a $650 \mathrm{~kg} / \mathrm{m}^{3}$, no qual este compreende partículas inferiores a $100 \mu \mathrm{m}$, e consistem de cimento (200 a $450 \mathrm{~kg} / \mathrm{m}^{3}$ ) e fílers minerais, sendo pozolânico ou naturais. A alta quantidade de finos é necessária para a redução do atrito entre os agregados graúdos.

Neste contexto, o trabalho teve como objetivo verificar como a redução do consumo de cimento pode influenciar o comportamento do CAA no estado fresco e endurecido. Ressalta-se a importância desta pesquisa, pois possibilita a obtenção de um CAA com economia de materiais o que se apresenta vantajoso tanto por questões econômicas como sustentáveis. Para a dosagem dos concretos estudados foi utilizado o método apresentado por GOMES [6]. Concretos contendo variados teores de cimento, abaixo do que é proposto pela literatura, com características autoadensáveis foram obtidos e posteriormente realizado sua caracterização mecânica. Os resultados mostraram que é possível obter CAABCC com quantidade de cimento menores do que usualmente é empregado, mantendo propriedades mecânicas desejáveis.

\subsection{Indicadores de baixo consumo de cimento}

Tradicionalmente pode-se expressar a relação entre a massa de cimento utilizada pelo volume de concreto produzido como um fator que mede o consumo de cimento naquela mistura. Em diversos países e no Brasil, é adotado o Sistema Internacional de medida que costumeiramente é apresentado em $\mathrm{kg} / \mathrm{m}^{3}$ [1]. Consumos tradicionais enquadram-se dentro do intervalo de 250 a $750 \mathrm{~kg} / \mathrm{m}^{3}$ [7]. O método adotado para expressar o consumo, não se relaciona com as características dos materiais envolvidos e nem do concreto resultante. Nem sempre um concreto com um consumo de cimento maior estará relacionado com uma resistência elevada.

Portanto, pode-se concluir que, um melhor indicativo de consumo seria aquele que relaciona a quantidade necessária de cimento para produzir certa quantidade de um determinado parâmetro, avaliando, assim, o desempenho obtido pela quantidade de cimento empregada. De acordo com REBMANN [2] pode-se expressar este consumo relativo matematicamente pela Equação 1:

$$
\mathrm{Cr}=\mathrm{C} / \mathrm{P}
$$

Sendo C o consumo de cimento e p o parâmetro escolhido.

Um dos principais parâmetros utilizados para retratar a eficiência estrutural do concreto é a resistência à compressão aos 28 dias. Desta maneira, um índice de consumo pode ser gerado levando em consideração a resistência obtida pelo concreto, à quantidade de cimento em um $\mathrm{m}^{3}$ de concreto necessário para se produzir 1 $\mathrm{MPa}$ de resistência à compressão. Por exemplo, um concreto convencional, apresentando um consumo de cimento igual a $400 \mathrm{~kg} / \mathrm{m}^{3}$, atingiu uma resistência à compressão de $35 \mathrm{MPa}$ aos 28 dias, assim, este concreto expressará um $\mathrm{Cr}$ igual a $11,43 \mathrm{~kg} / \mathrm{m}^{3}$ para atingir $1 \mathrm{MPa}$. Nem todas as situações em que se utiliza o parâmetro da resistência à compressão é essencial, por exemplo, em pisos, a resistência de maior interesse é a tração na flexão. Sendo assim, outros índices podem ser propostos, conforme o parâmetro desejado no momento.

Pode-se também variar este parâmetro, levando em consideração os diversos tipos de aglomerantes empregados na mistura, como sílica ativa, cinza volante, escória de alto forno etc. Como por exemplo BARBOZA [1], REBMANN [2], ALMEIDA FILHO [5], GOMES [6], PEREIRA[8], DAMINELI et al [9].

Um dos quesitos desta pesquisa é o aspecto ambiental, tendo o interesse em analisar a quantidade de $\mathrm{CO}_{2}$ emitido por MPa produzido. Este índice de consumo está relacionado à produção de clínquer, principalmente. Algumas características do concreto são consideradas, como por exemplo, o calor de hidratação, pois este aspecto está ligado diretamente à quantidade de clínquer utilizada na mistura. Ainda assim, pode-se propor um índice de $\mathrm{kg}$ de clínquer por MPa produzido por metro cúbico de concreto. E por fim, calcular a quantidade de $\mathrm{CO}_{2}$ emitido por MPa produzido.

\section{MATERIAIS E MÉTODOS}

\subsection{Materiais utilizados nas dosagens}

A escolha correta dos materiais pode ocasionar uma redução expressiva do consumo de cimento, no entanto, se isso levar à escolha de materiais que apresentam comportamento diferenciado, que geralmente não são encontrados com facilidade ou não estão disponíveis em diversas localidades, a aplicação em grande escala se inviabiliza. Desta forma, neste estudo optou-se por agregados que são encontrados atualmente no mercado na região 
metropolitana de Campinas, cidade de Engenheiro Coelho (SP), local de desenvolvimento da pesquisa e, o cimento e aditivo são facilmente encontrados comercialmente

\subsubsection{Cimento}

Foi utilizado apenas um tipo de cimento: CPII-Z-32, com massa específica de $3,02 \mathrm{~g} / \mathrm{cm}^{3}$, especificado pela ABNT NBR 11578 (1991) [10], e produzido pela empresa Votorantim. A razão da escolha quanto ao tipo de cimento, deve-se ao fato de ser um cimento muito utilizado na região no qual foi desenvolvida a pesquisa e de grande disponibilidade no mercado da construção civil.

\subsubsection{Agregado graúdo}

Foi selecionado um agregado natural britado de origem basáltica com dimensão máxima característica $9,5 \mathrm{~mm}$, classificado como 4,75/12,5 (d/D) e módulo de finura igual a 5,84 segundo a ABNT NBR NM 248 (2003) [11]. Os ensaios de caracterização contemplam a massa específica do agregado seco de $2,88 \mathrm{~g} / \mathrm{cm}^{3}$ segundo a ABNT NBR NM 53 (2009) [12], massa unitária em estado solto de 1,48 (g/ $\left.\mathrm{cm}^{3}\right)$, índice de vazios de 44,91\% de acordo com as diretrizes da ABNT NBR NM 45 (2006) [13], e absorção de água de 2,04\%.

\subsubsection{Agregado miúdo}

Foi selecionada uma areia, proveniente de cava de rio, com granulometria: areia muito fina, com dimensão máxima característica de 0,3 mm, módulo de finura 0,82, segundo a ABNT NBR NM 248 (2003) [11]. Os ensaios de caracterização contemplam a massa específica do agregado seco de $2,47 \mathrm{~g} / \mathrm{cm}^{3}$ de acordo com a ABNT NBR NM 52 (2009) [14], massa unitária em estado solto $1,44\left(\mathrm{~g} / \mathrm{cm}^{3}\right)$ e índice de volume de vazios de $36,45 \%$ de acordo com as diretrizes da ABNT NBR NM 45 (2006) [13].

\subsubsection{Adições minerais}

Foram selecionados duas adições minerais, uma sílica ativa e um fíler de calcário. A sílica ativa utilizada foi fabricada pela Tecnosil, possui massa específica de $2,22 \mathrm{~g} / \mathrm{cm}^{3}$, sua superfície específica (BET) é de aproximadamente $19000 \mathrm{~m}^{2} / \mathrm{kg}$ e tem teor de $\mathrm{SiO} 2>90 \%$. A sílica ativa utilizada tem retenção na peneira \#325 (abertura de $45 \mu \mathrm{m}$ ) inferior a 10\% [15]. A segunda adição, o fíler, é proveniente do calcário popularmente chamado de pó de calcário. As características do material fornecido pela empresa BRASIL MINAS indicam material de cor branca, com diâmetro médio de $45 \mu \mathrm{m}$. Sobre as características químicas indicam: $55,17 \%$ de $\mathrm{CaO}, 0,13 \%$ de $\mathrm{MgO}, 0,07 \%$ de $\mathrm{Fe}_{2} \mathrm{O}_{3}, 0,30 \%$ de $\mathrm{Al}_{2} \mathrm{O}_{3}$ e $0,77 \%$ de $\mathrm{SiO}_{2}[16]$.

\subsubsection{Superplastificante}

$\mathrm{O}$ aditivo superplastificante utilizado na pesquisa foi o ADVA ${ }^{\text {TM }}$ CAST 525 que se apresenta como aditivo de última geração baseado em policarboxilatos. De acordo com o fabricante, foi desenvolvido para extrema trabalhabilidade sem causar segregação no concreto, e foi especialmente formulado para produzir CAA. Apresentase na cor laranja, tem densidade média de $1,056 \mathrm{~g} / \mathrm{cm}^{3}$, segunda informação do fabricante. A porcentagem de aditivo está expressa em termos de aditivo total (sólidos + solventes) em relação a massa de cimento, no qual o teor de sólido do mesmo corresponde a 30\% [17].

\subsection{Dosagem do Concreto autoadensável com baixo consumo de cimento (CAABCC)}

Existem diversas propostas nomeadas para a determinação da composição do CAA, dentre elas podem ser citadas de SAAK et al. [7], ARIMA et al. [18], TUTIKIAN et al. [19], e da UPC proposto por GETTU e AGULLÓ [20]. A proposta adotada para o desenvolvimento do CAA foi baseada na pesquisa de GOMES [6] a qual se baseia no método de dosagem desenvolvido na UPC (Universidade Politécnica da Catalunha). Segundo a avaliação, o CAA pode ser determinado por meio de três etapas: pasta, agregado (esqueleto granular) e concreto.

O método da UPC é baseado na otimização separada da composição da pasta e do esqueleto granular dos agregados. A pasta é constituída de cimento, sílica ativa, fíler, água e superplastificante, enquanto o esqueleto granular é composto pela otimização entre agregado miúdo e graúdo de modo a se encontrar a maior compactação seca sem haver a necessidade de compactar os agregados; desse modo, há a redução de vazios no esqueleto granular [1].

\subsection{Ensaios preliminares}

Para a obtenção das dosagens empregadas foi preciso otimizar o emprego dos diversos materiais. A seguir são 
apresentados os resultados dos ensaios preliminares que permitiram definir os consumos dos diversos materiais empregados nos concretos definitivos.

\subsubsection{Esqueleto granular e empacotamento dos agregados}

A composição granular dos agregados (Figura 1) foi realizada com a intenção de se obter as relações entre os agregados, o teor de vazios existentes, a relação entre a quantidade de agregado miúdo e total dos agregados. Esse procedimento tem como base a ideia de que a combinação dos agregados com uma mínima quantidade de vazios levará a um menor consumo de pasta, consequentemente, menor consumo de cimento [6,21]. O empacotamento dos agregados deu-se a partir do ensaio de massa unitária no estado solto em diferentes proporções de agregados, de acordo com as diretrizes da ABNT NBR NM 45 (2006) [13] seguindo o método C da referida norma, sem compactação das camadas, pois segundo GOMES [6] não há sentido em compactar os grãos, uma vez que não existe adensamento no CAA.

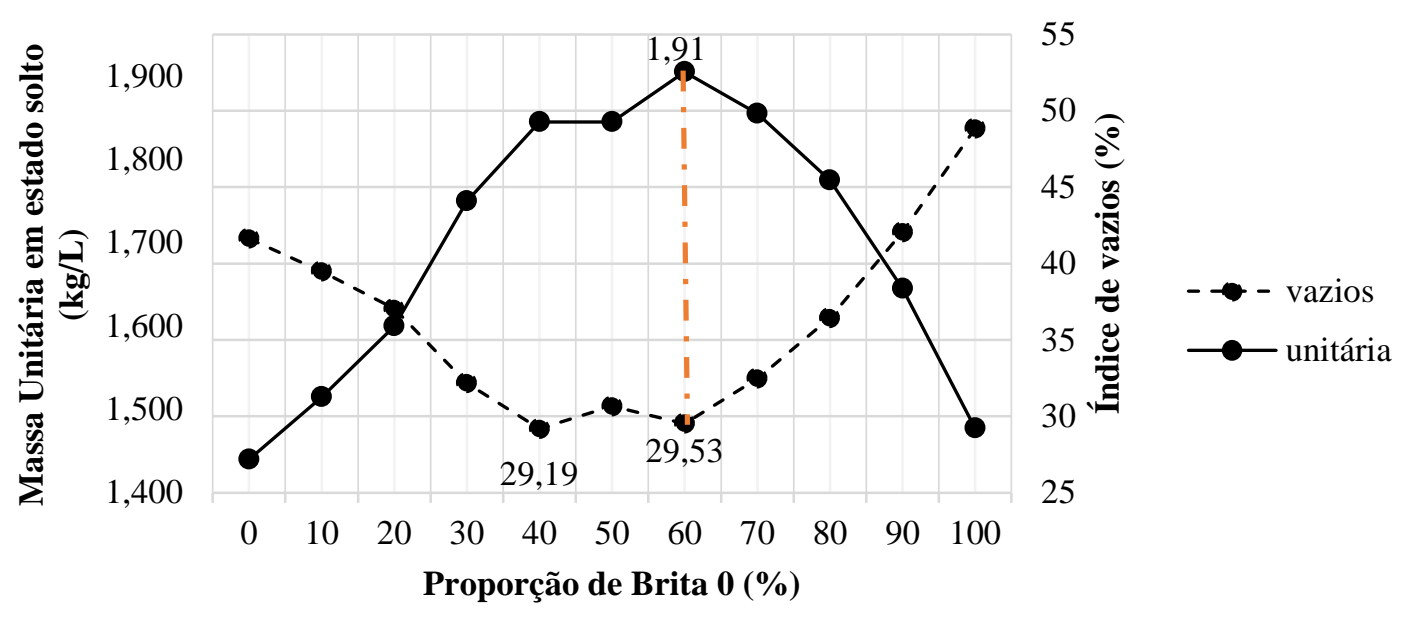

Figura 1: Empacotamento e índices de vazios dos agregados.

De acordo com os dados apresentados na Figura 1, nota-se um ponto ótimo com proporção de agregado graúdo em $40 \%$, sendo muito pequena a diferença para $60 \%$ de agregado graúdo. Optou-se por utilizar apenas $60 \%$ de agregado graúdo por resultar em uma massa unitária maior em relação ao ponto de $40 \%$ de agregado graúdo. No qual a proporção adotada de $60 \%$ de agregado graúdo e $40 \%$ de agregado miúdo corresponde ao mesmo valor proposto pelo parâmetro apresentado por GOMES [6] e GETTU e AGULLÓ [20] indicado na Figura 2. Portanto, a composição do esqueleto granular para essa condição tem $60 \%$ de brita e $40 \%$ de areia. Essa foi, portanto, a composição dos agregados fixada para todas as dosagens dos CAABCC.

Assim, para esta pesquisa também foi utilizado (Figura 2) como parâmetro o procedimento proposto por GOMES [6] e GETTU e AGULLÓ [20] para a determinação do teor ótimo de agregados levando em consideração que a relação AF/AT (Agregados finos/Agregado total) é igual a 47,5\% e AF/AG (Agregados finos/Agregado graúdo) igual a 95,0\% [22].

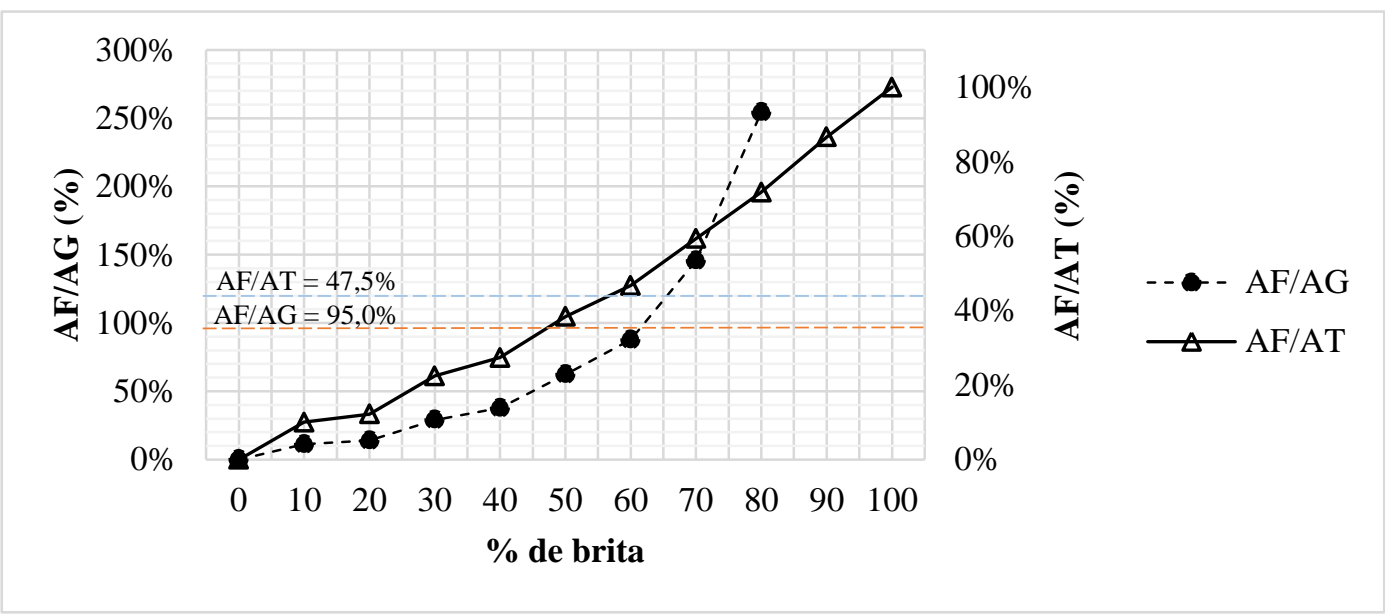

Figura 2: Empacotamento e índices de vazios dos agregados. 


\subsubsection{Avaliação do teor ótimo de aditivo - Etapa pasta}

Foi realizada a determinação do teor ótimo de aditivo superplastificante (ponto de saturação). No qual, foram utilizadas pastas de cimento, com adição de 10, 20, 30 e 40\% de fíler calcário, e 10\% sílica ativa, valor usualmente adotado por diversos pesquisadores , no qual os mesmos correspondem (GOMES [6], GETTU e AGUILLO [20], ALMEIDA FILHO [5], SANTOS [23] e BARBOZA [1]) e determinou-se o ponto de saturação do aditivo superplastificante utilizando dois métodos de ensaio: Funil Marsh para determinação do tempo de escoamento e o ensaio de miniabatimento para determinar o abatimento segundo a ABNT NBR 13276 (2002) [24] para um tempo de 10 minutos, com relação a/c de 0,4 .

São apresentados os resultados para o ensaio de miniabatimento para as variadas composições na Tabela 1 , ressalta-se que o tempo de espalhamento $\mathrm{T}_{115}$ por conta da alta fluidez da pasta pode apresentar erros em sua leitura.

Tabela 1: Resultados obtidos no ensaio de fluidez e miniabatimento para uma relação a/c 0,4 .

\begin{tabular}{l|c|c|c|c}
\hline PROPRIEDADES & \multicolumn{4}{|c}{ RESULTADOS OBTIDOS } \\
\hline SA/C (\%) & 10 & 10 & 10 & 10 \\
\hline FC/C (\%) & 10 & 20 & 30 & 40 \\
\hline SP/C (\%) - Saturação & 1,2 & 1,6 & 1,5 & 2 \\
\hline Espalhamento (mm) & 175,05 & 206,15 & 190,75 & 189,00 \\
\hline $\mathrm{T}_{115}$ & 2 & 1,5 & 1 & 2 \\
\hline Densidade da pasta (g/cm $\left.{ }^{3}\right)$ & 1,997 & 2,015 & 2,03 & 2,062 \\
\hline Sendo: SA/C a relação sílica ativa e cimento; FC/C a relação fíler \\
calcário e cimento e SP/C a relação superplastificante e cimento.
\end{tabular}

De acordo com GETTU e AGUILLÓ [20] e GOMES [6], os critérios para o teor ótimo de fíler na mistura, o diâmetro de espalhamento deve estar no intervalo de $18,0 \pm 1,0 \mathrm{~cm}$ e que o tempo $\mathrm{T}_{115}$ tem que estar no intervalo de $3 \pm 1$ segundos.

Tendo em vista que a maior composição estudada com $40 \%$ de adição de fíler de calcário obteve bons resultados nos ensaios, dentro do que é admitido de acordo com os autores citados, e que a pasta não apresentou segregação, optou-se por utilizar essa proporção, uma vez que o objetivo principal deste trabalho é a redução do consumo de cimento. Nota-se que a proporção escolhida apresentou a maior densidade.

O teor ótimo de superplastificante foi determinado mediante aos ensaios de funil Marsh. As curvas do "logaritmo do tempo de escoamento versus teor de aditivo" para a pasta de cimento produzida com relação $\mathrm{A} / \mathrm{C}=0,4, \mathrm{SA} / \mathrm{C}=0,10$ e FC/C 0,1, 0,2, 0,3 e 0,4 no tempo de 10 minutos são apresentadas na Figura 3.

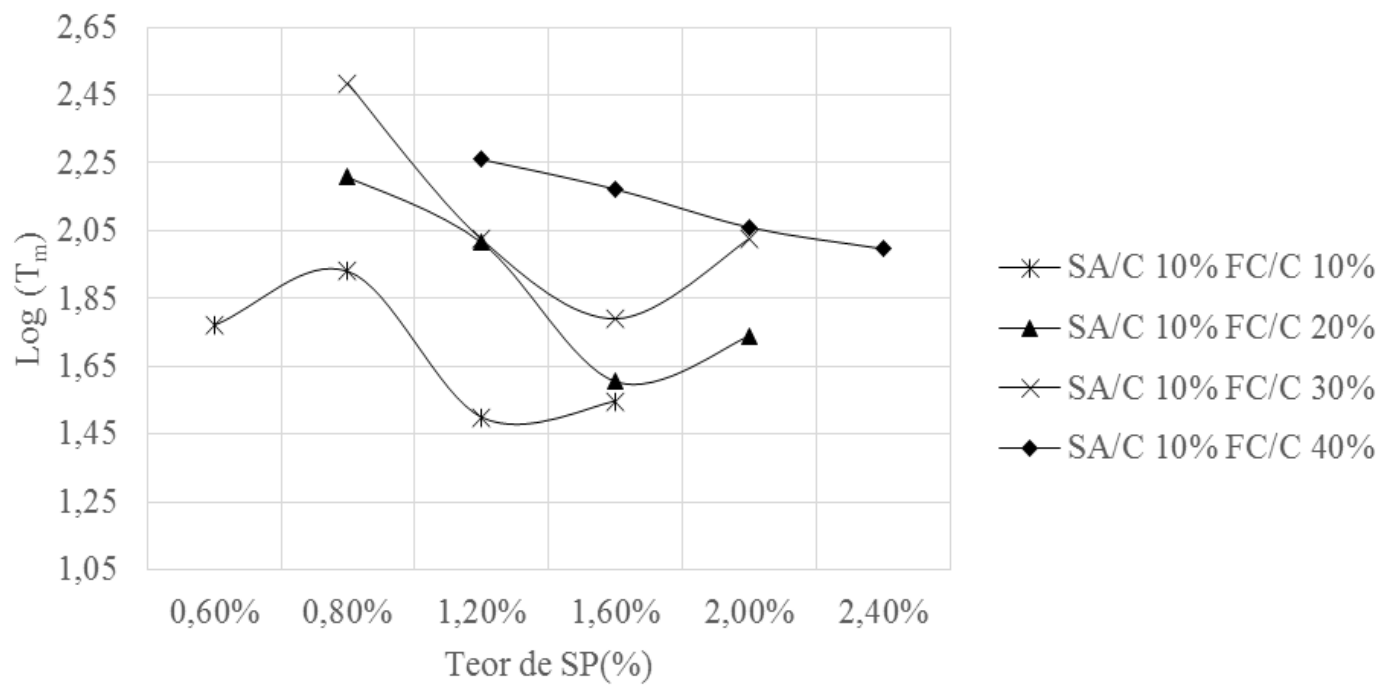

Figura 3: Variação do teor de superplastificante para a/c 0,4. 
Verificou-se, com o aumento da porcentagem de fíler calcário ocorria a perda de fluidez da pasta. Além disso, constatou-se que as pastas com maiores teores de finos apresentaram maiores tempos de fluxo, o que implica uma menor fluidez, em comparação com as pastas com menor relação FC/C.

Na Figura 4 é apresentado o teor ótimo de superplastificante encontrado para a pasta composta por $40 \%$ de fíler de calcário, composição escolhida para essa pesquisa. $\mathrm{O}$ valor foi obtido utilizando-se a curva para o tempo de 10 minutos.

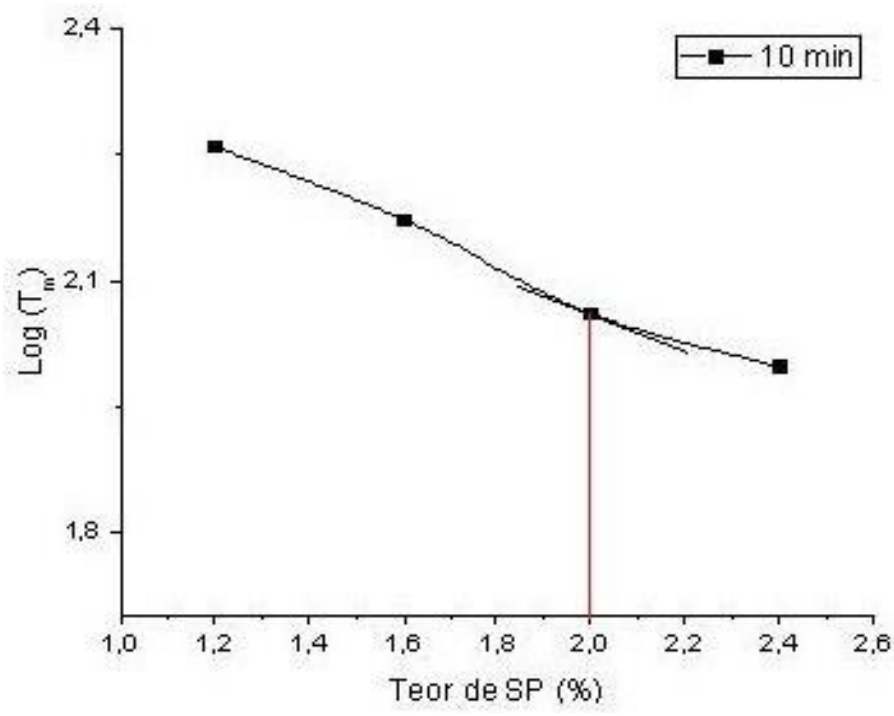

Figura 4: Curva para obtenção do teor ótimo de superplastificante para a/c 0,4.

Assim, a otimização das pastas de cimento definiu a matriz detalhada na Tabela 2 para os concretos autoadensáveis a serem produzidos.

Tabela 2: Resultados obtidos no ensaio de fluidez e miniabatimento para uma relação a/c 0,4.

\begin{tabular}{l|c}
\hline Cimento & CP II - Z -32 \\
\hline Teor de sílica & $10 \%$ \\
\hline Relação água/cimento & 0,40 \\
\hline Teor de superplastificante & $2,00 \%$ \\
\hline
\end{tabular}

O teor ótimo de superplastificante foi considerado para o tempo de 10 minutos após a mistura do cimento com água. No estudo de fluidez da pasta, a temperatura variou de 22 a $26^{\circ} \mathrm{C}$, com umidade relativa do ar entre 60 e $80 \%$, para todos os ensaios relacionados. Com isso, o teor de superplastificante (teores ótimos) foi de $2,006 \%$ para $\mathrm{FC} / \mathrm{C}=0,4$, adotando aproximadamente $2,00 \%$.

\subsection{Dosagens dos concretos autoadensáveis}

As dosagens foram desenvolvidas com base nos limites exigidos para atender a classe de agressividade ambiental tipo II, da ABNT NBR 12655 (2006) [25]. Esses limites constituem-se de consumo de cimento de, no mínimo $280 \mathrm{~kg} / \mathrm{m}^{3}$, relação água/cimento de no máximo, 0,6 e resistência à compressão aos 28 dias de, no mínimo, $30 \mathrm{MPa}$. No qual, estes parâmetros devem ser atendidos simultaneamente.

O cimento utilizado foi o mesmo das dosagens com baixo consumo de cimento, assim, como os outros materiais que irão compor o concreto de referência. Na Tabela 3 está apresentada a composição do concreto de referência (CR) e a composição dos concretos com baixo consumo de cimento (CAABCC) e na Tabela 4 alguns parâmetros destas mesmas dosagens. 
Tabela 3: Consumo de materiais por $\mathrm{m}^{3}$ de concreto $\left(\mathrm{kg} / \mathrm{m}^{3}\right)$

\begin{tabular}{l|c|c|c|c}
\hline MATERIAIS & CR & CAABCC1 & CAABCC2 & CAABCC3 \\
\hline Cimento (kg) & 434,06 & 358,10 & 347,25 & 325,54 \\
\hline Fíler Calcário (kg) & 173,62 & 143,24 & 138,90 & 130,22 \\
\hline Sílica Ativa (kg) & 43,41 & 35,81 & 34,72 & 32,55 \\
\hline Areia (kg) & 648,70 & 724,39 & 735,20 & 756,82 \\
\hline Brita (kg) & 973,06 & 1086,56 & 1102,80 & 1135,23 \\
\hline Superplastificante (g) & 868,00 & 716,00 & 694,00 & 651,00 \\
\hline Água (kg) & 173,62 & 143,24 & 138,90 & 130,22 \\
\hline
\end{tabular}

Tabela 4: Consumo de materiais por $\mathrm{m}^{3}$ de concreto $\left(\mathrm{kg} / \mathrm{m}^{3}\right)$

\begin{tabular}{l|c|c|c|c}
\hline PARÂMETRO & CR & CAABCC1 & CAABCC2 & CAABCC3 \\
\hline Relação água/aglomerantes & 0,363 & 0,363 & 0,363 & 0,363 \\
\hline Volume de pasta $(\%)$ & 40,00 & 33,00 & 32,00 & 30,00 \\
\hline Massa específica $\left(\mathrm{kg} / \mathrm{dm}^{3}\right)$ & 2,430 & 2,480 & 2,465 & 2,350 \\
\hline Consumo de cimento $\left(\mathrm{kg} / \mathrm{m}^{3}\right)$ & 434,06 & 358,10 & 347,25 & 325,54 \\
\hline Teor de superplastificante $(\%)$ & 2,00 & 2,00 & 2,00 & 2,00 \\
\hline
\end{tabular}

\section{RESULTADOS E DISCUSSÕES}

Nesta seção serão apresentados os resultados e análises de todo o estudo experimental, idealizado em concretos autoadensáveis com baixo consumo de cimento.

\subsection{Propriedades do concreto no estado fresco}

A mistura foi realizada em betoneira no modelo por gravidade com capacidade do tambor de 400 litros, capacidade de mistura 320 litros e rotação do tambor de $28 \mathrm{Rpm}$, seguindo a mesma ordem de mistura para todas as dosagens.

Tanto os concretos de referência quanto os de baixo consumo de cimento, foram misturados conforme procedimentos apresentado por GOMES [6] e ALMEIDA FILHO [5] na seguinte sequência:

- Agregado graúdo mais 3/4 da água total de amassamento (água para a pasta e água para saturar os agregados) e mistura-se por 30 segundos;

- Cimento, sílica ativa e o pó de calcário com o superplastificante e o restante da água e mistura por 120 segundos;

- E por fim, adiciona-se o agregado miúdo e mistura-se por 120 segundos.

A mistura ocorreu em sequência, suficiente para promover a homogeneização dos materiais presentes na betoneira, totalizando assim aproximadamente um tempo de mistura igual a 4 e $1 \frac{1}{2}$ minutos.

Finalizado e alcançada a trabalhabilidade esperada, as misturas foram submetidas aos ensaios de estado fresco segundo a ABNT NBR 15823 (2010) [26], sendo eles, caixa-L, Funil-V e Slump flow test. Em todos os ensaios realizados para caracterização dos concretos frescos, não se utilizou qualquer meio de adensamento, além do peso próprio do concreto.

Os dados que apontam as características dos concretos em análise no estado fresco são apresentados na Tabela 5, respectivamente, do concreto de referência e dos CAABCC.

Tabela 5: Caracterização dos concretos de referência e de baixo consumo de cimento no estado fresco

\begin{tabular}{c|c|c|c|c|c}
\hline PROPRIEDADES & $\begin{array}{c}\text { LIMITES ABNT } \\
\text { NBR 15823 [27] }\end{array}$ & CR & CAABCC1 & CAABCC2 & CAABCC3 \\
\hline Slump flow test, $\mathrm{d}_{\mathrm{f}}(\mathrm{mm})$ & $660 \leq \mathrm{d}_{\mathrm{f}} \leq 750$ & 815,00 & 732,50 & 760,00 & 667,50 \\
\hline $\mathrm{T}_{500}(\mathrm{~s})$ & $>2$ & $02 " 99$ & $06 ” 05$ & $06 " 23$ & 24 "49 \\
\hline Caixa L*, $\mathrm{H}_{2} / \mathrm{H}_{1}$ & $\geq 0,80$ & 0,81 & 0,81 & 0,93 & 0,82 \\
\hline
\end{tabular}




\begin{tabular}{|c|c|c|c|c|c|}
\hline Caixa $\mathrm{L}, \mathrm{t}_{20}(\mathrm{~s})$ & --- & $01 ” 20$ & $02 ” 24$ & 02”09 & $02 " 37$ \\
\hline Caixa $L, t_{40}(s)$ & --- & 02”08 & $08 " 95$ & $04 " 83$ & 08”01 \\
\hline Funil V, $\mathrm{t}_{\mathrm{V}}(\mathrm{s})$ & $9 \leq t_{v} \leq 25$ & 09"11 & $11 " 14$ & $10 " 92$ & $12 " 49$ \\
\hline
\end{tabular}

*Foram utilizados $4 \phi 12,5 \mathrm{~mm}$ espaçados de $3,2 \mathrm{~cm}$.

Todos os concretos ensaiados apresentaram fluidez elevada. Mesmo assim, apresentaram grande coesão, sem qualquer indício de segregação. Na Figura 5 são apresentadas algumas fotos dos concretos no estado fresco, evidenciando alta fluidez sem segregação, teor de argamassa adequado e bom aspecto de acabamento.

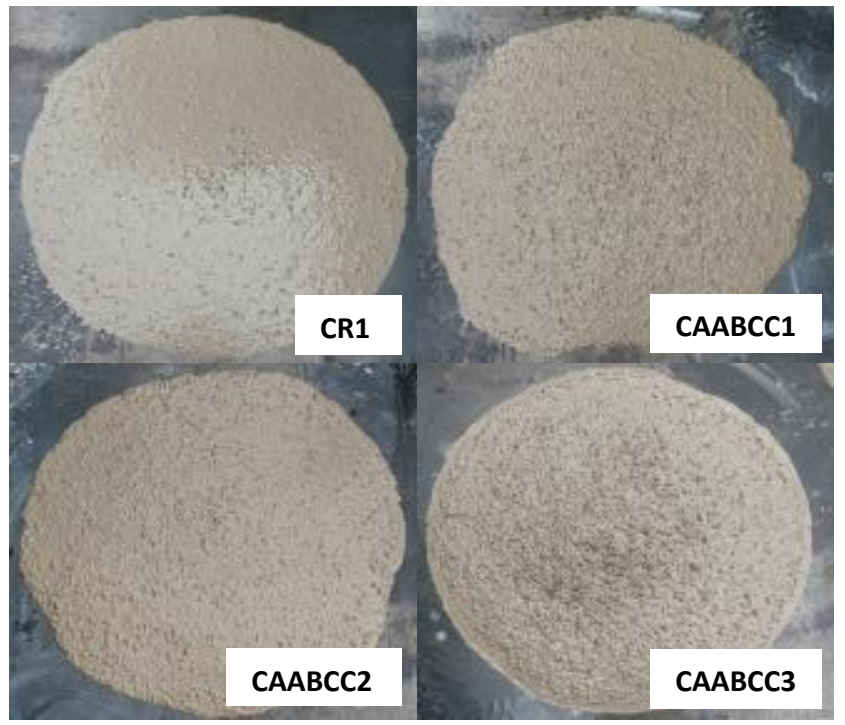

Figura 5: Espalhamento dos concretos analisados após o ensaio de slump flow test.

Deve-se justificar o espalhamento bem próximo do CAABCC3 ao limite mínimo apresentado por norma de $600 \mathrm{~mm}$. Devido ao baixo volume de pasta, consequentemente de cimento, decidiu-se não aumentar mais o consumo de água, pois as dosagens apresentavam indícios de início de exsudação. Este é um problema que se encontra ao utilizar consumos de cimento, e consequentemente de finos, muito baixos. Neste caso os valores de espalhamento encontrado não significaram qualquer prejuízo em termos de trabalhabilidade ou acabamento, mas, caso fosse necessário maior abatimento, uma alternativa seria realizar ajustes nos demais finos presentes, possivelmente com consumo maior de sílica e/ou pó de calcário. Vale dizer que a ocorrência de exsudação é sempre indesejável, devendo ser evitada, pois proporciona perda de resistência à compressão.

\subsection{Propriedades do concreto no estado endurecido}

Para a caracterização mecânica, foram moldados corpos de prova cilíndricos com dimensões de 10 x $20 \mathrm{~cm}$ para a realização dos ensaios de resistência à compressão axial nas idades de 3, 7 e 28 dias.

O ensaio de compressão em corpos de prova cilíndricos, de diâmetro de 10 x $20 \mathrm{~cm}$, foi realizado de acordo com a ABNT NBR 5739 (2007) [27], com velocidade de carregamento constante e igual de 0,5 $\mathrm{MPa} / \mathrm{s}$. A Figura 4.18 ilustra um corpo de prova sendo submetido ao ensaio de compressão.

São apresentados, na (Figura 6), os resultados médios obtidos para os ensaios de resistência à compressão realizados para os concretos, cujas propriedades no estado fresco se mostraram satisfatórias à autoadensabilidade. 


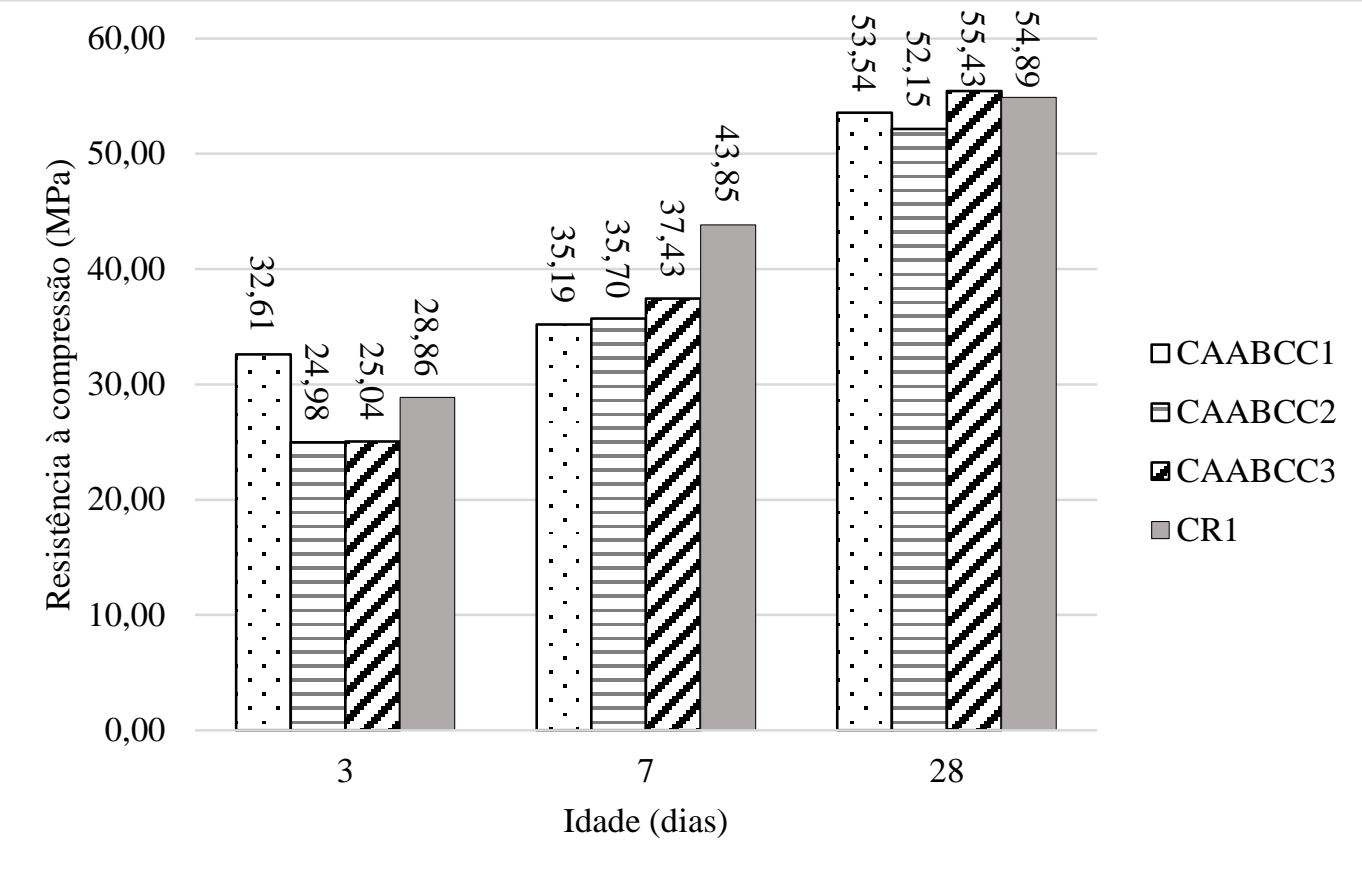

Figura 6: Resultados da resistência à compressão média dos concretos autoadensáveis.

Fez-se também, uma análise estatística básica dos resultados obtidos, para avaliar a dispersão destes, nas tabelas 6 e 7 são apresentados estes valores.

Tabela 6: Características estatísticas da resistência à compressão do concreto de referência.

\begin{tabular}{c|c|c|c}
\hline RESISTÊNCIA À COMPRESSÃO & 3 DIAS & 7 DIAS & 28 DIAS \\
\hline Média (MPa) & 32,61 & 43,85 & 54,89 \\
\hline Desvio Padrão $(\mathrm{MPa})$ & 0,78 & 1,32 & 0,98 \\
\hline C.V (\%) & 2,41 & 3,02 & 1,78 \\
\hline
\end{tabular}

Tabela 7: Características estatísticas da resistência à compressão dos concretos com consumo de cimento reduzido

\begin{tabular}{c|c|c|c|c|c|c|c|c|c}
\hline \multirow{2}{*}{$\begin{array}{c}\text { RESISTÊNCIA À } \\
\text { COMPRESSÃO }\end{array}$} & \multicolumn{3}{|c|}{ CAABCC 1 } & \multicolumn{3}{c|}{ CAABCC 2 } & \multicolumn{3}{c}{ CAABCC 3 } \\
\cline { 2 - 11 } & 3 DIAS & 7 DIAS & 28 DIAS & 3 DIAS & 7 DIAS & 28 DIAS & 3 DIAS & 7 DIAS & 28 DIAS \\
\hline Média (MPa) & 23,50 & 35,18 & 53,54 & 24,98 & 35,69 & 52,15 & 25,04 & 37,43 & 55,43 \\
\hline $\begin{array}{c}\text { Desvio Padrão } \\
(\mathrm{MPa})\end{array}$ & 0,98 & 1,17 & 1,25 & 0,59 & 0,03 & 1,90 & 0,29 & 1,42 & 1,36 \\
\hline C.V (\%) & 4,16 & 3,32 & 2,34 & 2,34 & 0,09 & 3,64 & 1,18 & 3,80 & 2,45 \\
\hline
\end{tabular}

Nota-se que em ambos os concretos, a resistência à compressão aumentou com o tempo. É possível verificar, também, que o CAABCC3 que apresenta o menor volume de pasta, consequentemente menor consumo de cimento, apresentou resistência superior ao demais concretos, inclusive o de referência aos 28 dias. Acredita-se que esta verificação é gerada pela à maior compacidade conferida à pasta pelo aumento do consumo de areia muito fina na mistura.

Segundo OKAMURA [28]; DOMONE [29] e KIN et al. [30], quanto mais finas forem as partículas de agregado miúdo, maior a sua capacidade de preenchimento de poros não preenchidos com as partículas de agregado graúdo, aumentando desta forma, a densidade da mistura e consequentemente a sua resistência à compressão. Além disso, uma melhor hidratação das partículas de cimento, devido a maior retenção de água. Esta afirmação está de acordo com o que é apresentado por REPETTE [31], as quais dizem que tanto a hidratação do cimento quanto a aderência do CAA nas armaduras são melhoradas em função da maior retenção de água que este tipo de mistura pode proporcionar. 
O comportamento mecânico de concretos com elevados teores de adições minerais é condicionada ao processo de hidratação da mistura cimento-adições, pois as adições minerais, em substituição do cimento, podem gerar uma redução da resistência inicial, afetando a resistência aos 28 dias, em comparação com concretos sem adições minerais [32] [33].No entanto, ao longo do tempo, é expectável que se verifique alguma recuperação da resistência devido à lentidão da reação pozolânica das adições que é decorrente da disponibilização de $\mathrm{CH}$ proveniente da reação de hidratação do cimento [34]. Ainda Ahmaruzzaman [35] afirma, que em concretos com altos teores de adições pode ocorrer a ausência de $\mathrm{CH}$ em idades próximas ou acima de 28 dias, pois todo o $\mathrm{CH}$ produzido pela hidratação do cimento seja consumido por parte das adições minerais logo até aos 28 dias e, desta forma, as adições minerais colocadas em excesso podem não estar reagindo quimicamente, funcionando apenas como material de enchimento.

\subsection{Indicadores de baixo consumo de cimento}

Geralmente a resistência à compressão é considerada como a propriedade mais importante do concreto, sendo quase que invariavelmente um elemento essencial do projeto estrutural. Mesmo no CAA, no qual as propriedades no estado fresco são tão importantes, o conhecimento da resistência à compressão se faz necessária para a comparação do seu custo de produção em relação a um concreto convencional. Deste modo, nesta pesquisa foi utilizada a resistência à compressão como parâmetro principal de avaliação de desempenho. Este parâmetro foi avaliado em face do consumo de cimento, consumo total de materiais cimentícios, consumo de clínquer e emissão aproximada de $\mathrm{CO}_{2}$.

Na Tabela 8 estão resumidos os consumos para volume de $1 \mathrm{~m}^{3}$ das diversas dosagens. O teor de clínquer no CP II - Z - 32 é de 76\% de acordo com as informações disponibilizadas pelo fabricante VOTORANTIM [36]. De acordo com PEREIRA [8], a emissão de $\mathrm{CO}_{2}$ para cimento $\mathrm{CP}$ II - Z - 32 foi estimado em $0,84 \mathrm{~kg}$ de $\mathrm{CO}_{2}$ por kg de clínquer.

Tabela 8: Consumos de cimento, material cimentício, clínquer e emissão de $\mathrm{CO}_{2}$ para as dosagens.

\begin{tabular}{c|c|c|c|c}
\hline \multirow{2}{*}{ CONSUMOS } & \multicolumn{4}{|c}{ DOSAGENS } \\
\cline { 2 - 5 } & CR1 & CAABCC 1 & CAABCC 2 & CAABCC 3 \\
\hline Cimento & 434,06 & 358,10 & 347,25 & 325,54 \\
\hline Cimentícios & 477,47 & 393,91 & 381,97 & 358,09 \\
\hline Clínquer & 329,86 & 272,16 & 263,91 & 247,41 \\
\hline $\mathrm{CO}_{2}$ & 277,08 & 228,61 & 221,68 & 207,82 \\
\hline
\end{tabular}

A partir destes consumos, e utilizando os valores de resistência à compressão obtidos nas idades analisadas, pode-se calcular os consumos relativos para cada idade (Tabela 9).

Tabela 9: Consumo de cimento, material cimentício, clínquer e emissão de $\mathrm{CO}_{2}$ para as quatro dosagens analisadas relativos à resistência à compressão aos $28 \operatorname{dias~}\left(\mathrm{kg} / \mathrm{m}^{3} / \mathrm{MPa}\right)$.

\begin{tabular}{c|c|c|c|c}
\hline CONSUMO & CR1 & CAABCC 1 & CAABCC 2 & CAABCC 3 \\
\hline Cimento & 7,91 & 6,69 & 6,66 & 5,87 \\
\hline Cimentícios & 8,70 & 7,36 & 7,32 & 6,46 \\
\hline Clínquer & 6,00 & 5,08 & 5,06 & 4,46 \\
\hline $\mathrm{CO}_{2}$ & 5,05 & 4,27 & 4,25 & 3,75 \\
\hline
\end{tabular}

Notou-se em geral que os consumos relativos de cimento aos 28 dias ficaram entre de 5,87 a 6,69 $\mathrm{kg} / \mathrm{m}^{3}$ por MPa para os concretos com baixo consumo de cimento, enquanto o concreto de referência 7,91 $\mathrm{kg} / \mathrm{m}^{3}$ por MPa. Comparando com os resultados obtidos por DAMINELI et al. [9], que consideraram o total de materiais cimentícios, obtém-se na Figura 7. Notou-se o bom resultado obtido, principalmente as dosagens com baixo consumo de cimento estando abaixo do limite inferior da grande maioria dos resultados coletados. Já o concreto de referência ficou contido dentro da grande nuvem média. 
Dados Nacionais

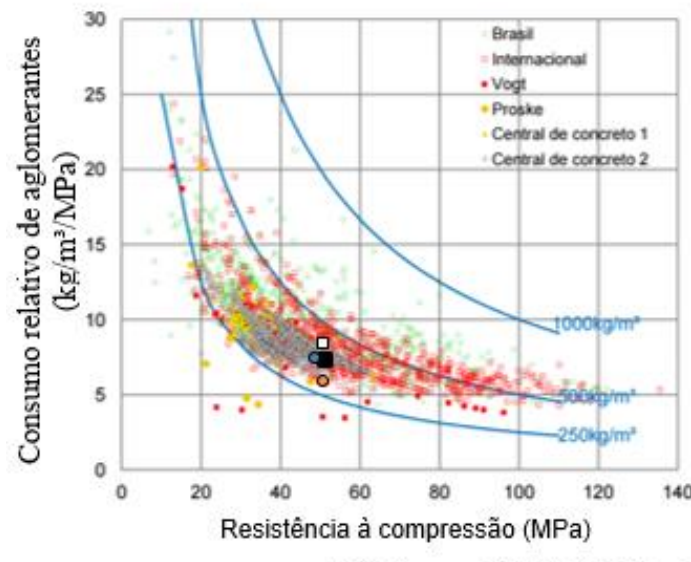

口CR1
Dados Internacionais

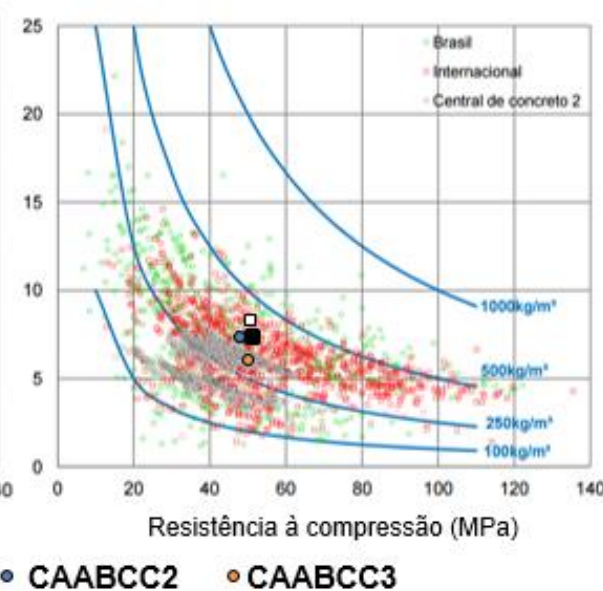

Figura 7: Relação entre o consumo de aglomerantes e resistência à compressão aos 28 dias (DAMINELLI et al. [9]).

De modo geral, os concretos em estudo apresentaram ótimo desempenho, tanto em termos de consumo de material cimentício como em termos de emissão de $\mathrm{CO}_{2}$, além do consumo total de cimento reduzido.

A Figura 8 apresenta a comparação do consumo de cimento entre todos os concretos dosados, bem como a redução percentual deste consumo por parte dos CAABCC, referentes à resistência à compressão aos 28 dias.

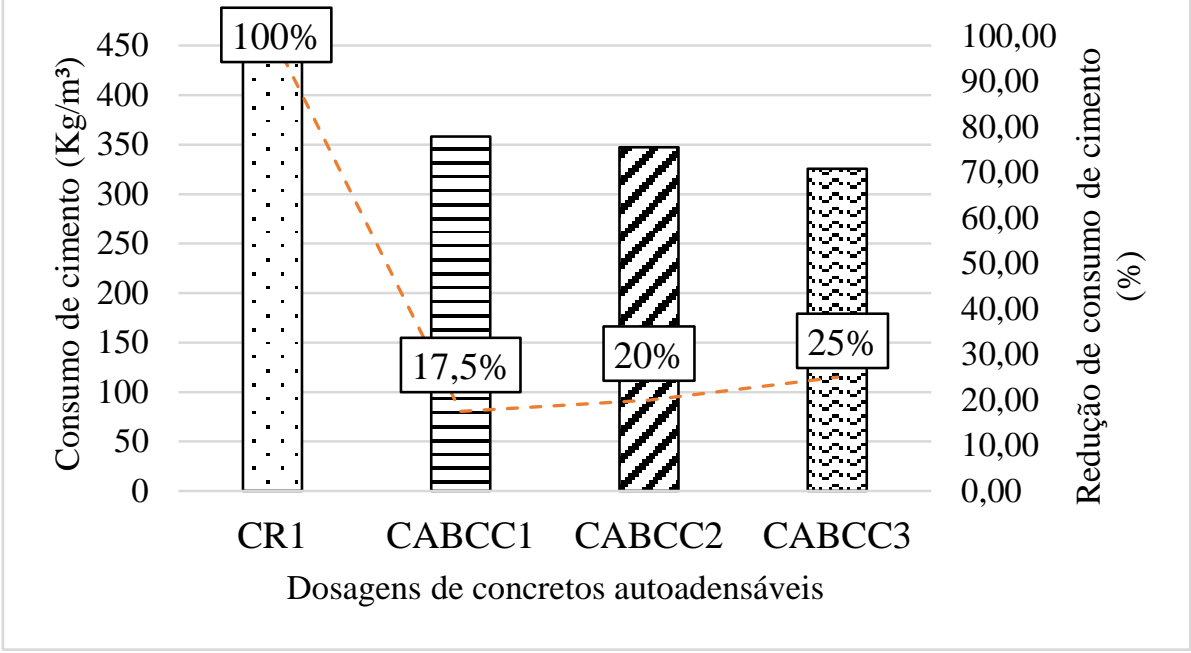

Figura 8: Consumo de cimento estre os concretos produzidos e a redução percentual deste consumo dos CAA.

Observou-se que foi possível atingir uma redução de $25 \%$ do consumo de cimento (CAABCC3) em comparação ao concreto de referência (CR). No qual o CR apresentou um consumo de cimento de 434,06 $\mathrm{kg} / \mathrm{m}^{3}$ e $325,54 \mathrm{~kg} / \mathrm{m}^{3}$ para o CAABCC 3 , respectivamente, apresentando aproximadamente uma redução de $110 \mathrm{~kg} / \mathrm{m}^{3}$.

\subsection{Análise da viabilidade econômica}

Para verificar a viabilidade econômica da elaboração de concretos do tipo propostos neste trabalho inclui-se aqui esta breve avaliação de custos. Esta verificação de delimita ao custo de materiais, considerando que não sejam necessárias grandes alterações de custos na mão de obra e equipamentos para produção. Na Tabela 10 são apresentados os custos unitários dos diversos materiais utilizados. Trataram-se de valores aproximados praticados na região metropolitana de Campinas, cidade de Engenheiro Coelho - SP para ano de 2017, se- 
gundo os fornecedores.

Tabela 10: Custos unitários dos materiais empregados $(\mathrm{R} \$ / \mathrm{kg})$

\begin{tabular}{c|c|c|c|c|c}
\hline CIMENTO & SÍLICA ATIVA & FÍLER CALCÁRIO & AREIA & BRITA & ADITIVO \\
\hline 0,40 & 1,15 & 0,75 & 0,058 & 0,064 & 7,00 \\
\hline
\end{tabular}

Na Tabela 11 estão calculados os custos por $\mathrm{m}^{3}$ de cada uma das dosagens adquiridas. Na terceira linha desta tabela para a avaliação do custo benefício, são apresentados os custos relativos, tomando como base a resistência à compressão média alcançada aos 28 dias por cada dosagem desenvolvida.

Tabela 11: Consumo de cimento, material cimentício, clínquer e emissão de $\mathrm{CO}_{2}$ para as quatro dosagens analisadas relativos à resistência à compressão aos $28 \operatorname{dias~}\left(\mathrm{kg} \cdot \mathrm{m}^{-3} / \mathrm{MPa}\right)$.

\begin{tabular}{c|c|c|c|c}
\hline PARÂMETROS & CR & CAABCC 1 & CAABCC 2 & CAABCC 3 \\
\hline Custos Materiais $\left(\mathrm{R} \$ \mathrm{~m}^{3}\right)$ & 459,74 & 408,42 & 401,08 & 386,42 \\
\hline Resistência média 28 dias $(\mathrm{MPa})$ & 54,89 & 53,54 & 52,15 & 55,43 \\
\hline Custo relativo $\left(\mathrm{R} \$ \cdot \mathrm{m}^{-3} / \mathrm{MPa}\right)$ & 8,38 & 7,63 & 7,69 & 6,97 \\
\hline
\end{tabular}

Constata-se que correu uma diminuição de custo ao reduzir o consumo de cimento, cujo percentual de redução foi de aproximadamente de 16\%, comparando o concreto de referência e o CAABCC de menor custo, apresentando uma diferença de $\mathrm{R} \$ 73,32$ por metro cúbico. Conclui-se que a viabilidade econômica, uma vez que o custo-benefício é mais baixo em todos os concretos com baixo consumo de cimento.

Deve-se lembrar que os valores aqui apresentados são apenas indicativos, podendo as conclusões variarem com base no custo de cada material nas diversas localidades do Brasil. Também algumas variações nas propriedades dos materiais podem gerar um resultado em desempenho diferenciado. Assim sendo, para que possa ser possível reduzir o custo total dos concretos de baixo consumo, realizando estudos de otimização de consumo, principalmente dos itens mais caros como fíler, adições minerais e aditivos. Alterações nas proporções destes materiais influenciam tanto o custo total como o desempenho, de modo que o estudo de otimização de custo se faz necessário para atingir menores custos totais e relativos. Por fim, para uma análise mais conclusiva e completa, seria preciso desenvolver estudo de campo para observar a viabilidade de produção, lançamento e aplicação deste concreto, a fim de obter dados mais completos de custos, incluindo os custos de equipamentos e mão de obra para produção e aplicação do concreto.

\section{CONCLUSÕES}

Baseado nos resultados desta pesquisa, conclui-se que mesmo tendo uma redução do consumo de cimento de até $25 \%$ para o CAABCC 3 com $30 \%$ do volume de pasta, de um modo geral as quedas no desenvolvimento da resistência mecânica aos 28 dias dos concretos com baixo consumo de cimento foram inferiores a redução do consumo de cimento em função de menores volumes de pasta.

No caso específico do CAABCC 3, a redução do volume de pasta ocasionou a redução do consumo de cimento concomitantemente com a elevação da resistência mecânica à compressão, denotando que para volumes de pasta próximos ao índice de vazios ótimo obtido na fase do empacotamento de agregados, o incremento do volume de agregados proporcionado pela queda de volume de pasta, confere uma microestrutura do concreto com menor porosidade, o que corrobora para ganhos de resistência.

Em termos de viabilidade econômica, mesmo que a inferência do desempenho técnico do CAABCC tenho sido superior do que os termos econômicos, a minimização da quantidade de materiais com custo de maior magnitude (cimento e fíler calcário) e simultaneamente, de uso significativo na composição da dosagem (agregados graúdos e miúdos), proporcionou aos CAABCC um menor custo unitário do que ao CR 1.

Quanto ao viés ambiental, embora a queda de até $25 \%$ no consumo de cimento implique necessariamente em uma redução equivalente na emissão de $\mathrm{CO}_{2}$, o fato do cimento escolhido apresentar elevados índices de clínquer em sua composição (95\%), caracteriza que o cimento selecionado não é aconselhável em termos de sustentabilidade.

Portanto, é conclusivo que para os resultados apresentados nesta pesquisa, todos os CAABCC dosados possuíram superior desempenho técnico $\left(\mathrm{kg} \cdot \mathrm{m}^{-3} / \mathrm{MPa}\right)$ e financeiro $\left(\mathrm{R} \$ \cdot \mathrm{m}^{-3} / \mathrm{MPa}\right)$ do que o concreto CAA de referência, atendendo simultaneamente as exigências do CAA para o estado fresco e endurecido, e comprovando desta forma, a viabilidade econômica do CAABCC. 


\section{BIBLIOGRAFIA}

[1] BARBOZA, L.S., Estudo sobre o impacto da redução de consumo de cimento na resistência à compressão do concreto autoadensável, Dissertação de M.Sc, PPGECiv/UFSCar, São Carlos, SP, Brasil, 2016.

[2] REBMANN, M.S., Durabilidade de concretos estruturais com baixo consumo de cimento Portland e alta resistência, Dissertação de M.Sc, EESC/USP, São Carlos, SP, Brasil, 2011.

[3] MEHTA, P.K., MONTEIRO, P. J. M., Concreto: microestrutura, propriedades e materiais, $2^{\mathrm{a}}$ ed., São Paulo, IBRACON, 2014.

[4] EFNARC, European federation of specialist construction chemicals and concrete system. Specification and guidelines for self-compacting concrete. s.l.: EFNARC, 2002.

[5] ALMEIDA FILHO, F.M., Contribuição ao estudo da aderência entre barras de aço e concretos autoadensáveis, Tese de D.Sc, EESC/USP, São Carlos, SP, Brasil, 2006.

[6] GOMES, P.C.C., Optimization and characterization of high-strength selfcompacting concrete, Tese de D.Sc, UPC, Catalunya, Barcelona, 2002.

[7] SAAK, A. W., JENNINGS, H. M., SHAH, S. P., "New methodology for designing self-compacting concrete", ACI Materials journal, v. 98, n. 6, pp. 429-439, Nov.-Dec. 2001.

[8] PEREIRA, T. A. C., Concreto autoadensável, de alta resistência, com baixo consume de cimento Portland e com adições de fibras de lã ou rocha ou poliamida, Dissertação de M.Sc, EESC/USP, São Carlos, SP, Brasil, 2010.

[9] DAMINELI, B. L., KEMEID, F. M., AGUIAR, P. S., et al., "Measuring the eco-efficiency of cement use", Cement and Concrete Composites, v. 32, n. 8, pp. 555-562, set. 2010.

[10] ASSOCIAÇÃO BRASILEIRA DE NORMAS E TÉCNICAS. NBR 11578: Cimento Portland Composto. Rio de Janeiro, 1991.

[11] ASSOCIAÇÃO BRASILEIRA DE NORMAS E TÉCNICAS. NBR NM 248: Agregados: determinação da composição granulométrica. Rio de Janeiro, 2003.

[12] ASSOCIAÇÃO BRASILEIRA DE NORMAS E TÉCNICAS. NBR NM 53: Agregado graúdo: determinação da massa específica, massa específica aparente e absorção de água. Rio de Janeiro, 2009.

[13] ASSOCIAÇÃO BRASILEIRA DE NORMAS E TÉCNICAS. NBR NM 45: Agregados: determinação da massa unitária e do volume de vazios. Rio de Janeiro, 2009.

[14] ASSOCIAÇÃO BRASILEIRA DE NORMAS E TÉCNICAS. NBR NM 52: Agregados miúdo: determinação da massa específica e massa específica aparente. Rio de Janeiro, 2009.

[15] Sílica ativa (Ficha técnica do produto), TECNOSIL, http://www.tecnosilbr.com.br/produtos. Acessado em abril de 2017.

[16] Pó de calcário (Ficha técnica do produto), BRASIL MINAS, http://www.brasilminas.net/calcario. Acessado em abril de 2017.

[17] Aditivos para concreto, GRACE, https://www.aecweb.com.br/cls/catalogos/grace/adva_cast525.pdf. Acessado em abril de 2017.

[18] ARIMA, H., SADAKANE, M., AYANO. T., et al., "Experimental study on stable production of selfcompacting high performance concrete", In: Concrete structures in the 21 st century: Proceedings of the first FIB congresso, [CD-ROM], Osaka, Japão, Março. 2002.

[19] TUTIKIAN, B.F., Método para dosagem de concretos autoadensáveis, Dissertação de M.Sc, UFRGS, RS, Brasil, 2004.

[20] GETTU, R., AGULLÓSHAH, L., "Estado del hormigón autocompactable y su caracterización (parte I)", Cemento Hormigón, n. 861, pp. 50-67, 2004.

[21] GOLTERMANN, P., JOHANSEN, V., PALBOL, L., "Packing of aggregates: an alternative tool to determine the optimal mix", ACI Materials journal, n. 94, pp. 435-443, Nov. 1997.

[22] SU, N., HSU, K.; CHAI, H., "A simple Mix Design Method for Self-Compacting Concrete", Cement and Concrete Research, n. 31, pp. 1799-1807, Dec. 2001.

[23] SANTOS, R. F. C. Estudo de concretos autoadensáveis, com aditivo incorporador de ar, utilizados na produção de paredes de concreto armado moldadas no local, Dissertação M.Sc. - Departamento de Tecnologia em construção de edifícios, Instituto de Pesquisas Tecnológicas do Estado de São Paulo, São Paulo, 2012. 
[24] ASSOCIAÇÃO BRASILEIRA DE NORMAS E TÉCNICAS. NBR 13276: Argamassa para assentamento e revestimento de paredes e tetos - Preparo da mistura e determinação do índice de consiostência. Rio de Janeiro, 2002.

[25] ASSOCIAÇÃO BRASILEIRA DE NORMAS E TÉCNICAS. NBR 12655: Concreto de Cimento Portland: preparo, controle e recebimento: procedimento. Rio de Janeiro, 2015.

[26] ASSOCIAÇÃO BRASILEIRA DE NORMAS E TÉCNICAS. NBR 15823: Concreto autoadensável. Rio de Janeiro, 2010.

[27] ASSOCIAÇÃO BRASILEIRA DE NORMAS E TÉCNICAS. NBR 5739: Concreto -Ensaio de compressão de corpos de prova cilíndricos. Rio de Janeiro, 2007.

[28] OKAMURA, T., "Self-compacting high-performance concrete", Concrete International, v. 19, n. 7, pp. 50-54, 1997.

[29] DOMONE, P. L., Self-compacting concrete: An analysis of 11 years of case studies, In: Sciencedirect http://www.sciencedirect.com.br, 2005.

[30] KIM, D. S., SEO, W. S., LEE, K. M., "Method for nondestructive evaluation of concrete structure", Concrete International, v. 39, pp. 143-154, 2006.

[31] REPETTE, W.L. "Concreto autoadensável”, In: ISAIA, G. C. (ed), Concreto: Ensino, pesquisa e realizações, led., cápitulo 43, São Paulo, Brasil, IBRACON, 2005.

[32] CAMÕES, A., "Durability of high volume fly ash concrete", In: International RILEM workshop on performance based evaluation and indicators for concrete durability, Madrid, Spain, 19-21 march, pp. 311-318, 2006.

[33] ŞAHMARAN, M., YAMAN, İ.Ö., TOKYAY, M., "Transport and mechanical properties of self consolidating concrete with high volume fly ash", Cement and concrete composites, v. 31, n. 2, pp. 99-106, 2009.

[34] MALHOTRA, V., High-Performance High-Volume Fly Ash Concrete. Concrete International. ACI. v.24, n.7, pp. 30-34, 2002.

[35] AHMARUZZAMAN, M., "A review on the utilization of fly ash", Progress in Energy and Combustion Science, v.36, n. 3, pp. 327-363, 2010.

[36] Cimento Portland Composto (Ficha técnica do produto), VOTORANTIM, http://www.votorantimcimentos.com.br/hotsites/cimento/base.htm. Acessado em junho de 2017. 